\title{
RETRACTED ARTICLE: A 2-D DIGE analysis of simulated acid rain-responsive proteins in Pinus massoniana needles
}

Ting-Wu Liu $\cdot$ Wen-Jun Hu $\cdot$ Xiao-Qin Wang

Li Niu $\cdot$ Bin Fu $\cdot$ Fei-Hua Wu $\cdot$ Zhen-Ming Pei •

Hai-Lei Zheng

Received: 11 February 2012/Revised: 8 June 2012/ Accepted: 14 June 2012/Published online: 1 July 2012

(c) Springer-Verlag 2012

This article has been retracted due to disputed authorship.

T.-W. Liu · W.-J. Hu · X.-Q. Wang · L. Niu ·

B. Fu · F.-H. Wu · Z.-M. Pei · H.-L. Zheng ( $\varangle)$

Key Laboratory for Coastal and Wetland Ecosystem of Ministry

of Education, College of the Environment and Ecology,

Xiamen University, Xiamen 361005, Fujian,

People's Republic of China

e-mail: zhenghl@xmu.edu.cn

Z.-M. Pei

Department of Biology, Duke University,

Durham, NC 27708, USA

H.-L. Zheng

State Key Laboratory of Marine Environmental Science,

Xiamen University, Xiamen 361005, Fujian,

People's Republic of China 